\title{
Genome analysis and its significance in four unicellular algae, Cyanidioschyzon merolae, Ostreococcus tauri, Chlamydomonas reinhardtii, and Thalassiosira pseudonana
}

\author{
Osami Misumi · Yamato Yoshida $\cdot$ Keiji Nishida · Takayuki Fujiwara • \\ Takayuki Sakajiri · Syunsuke Hirooka · Yoshiki Nishimura · Tsuneyoshi Kuroiwa
}

Published online: 5 March 2008

(C) The Botanical Society of Japan and Springer 2008

\section{Erratum to: J Plant Res (2008) 121:3-17 \\ DOI 10.1007/s10265-007-0133-9}

In the above-mentioned article, the genus name Cyanidioschyzon was misspelled as Cyanidioshyzon in the title of the article, line 7 of the abstract, and lines 7 and 8 of the introduction section. The corrected article title is as given above, and the corrected sentences in the abstract and introduction are as follows.

In the abstract:

Whole genomes of the red alga Cyanidioschyzon merolae, the green algae Ostreococcus tauri and Chlamydomonas reinhardtii, and the diatom Thalassiosira pseudonana have been sequenced.

In the introduction:

Cyanidioschyzon merolae is a unicellular red alga which lives in extreme environments, such as those at high temperatures or that are strongly acidic (De Luca et al. 1978).

The online version of the original article can be found under doi:10.1007/s10265-007-0133-9.

O. Misumi $\cdot$ K. Nishida $\cdot$ T. Fujiwara $\cdot$ T. Sakajiri ·

S. Hirooka $\cdot$ T. Kuroiwa

Department of Life Science, Graduate School of Science,

Rikkyo University, Tokyo 171-8501, Japan

Y. Yoshida

Department of Integrated Biosciences,

Graduate School of Frontier Sciences,

The University of Tokyo, Chiba 277-8562, Japan

\section{Y. Nishimura}

Institute of Molecular and Cellular Biosciences,

The University of Tokyo, Tokyo 113-0032, Japan

T. Kuroiwa $(\bowtie)$

Research Information Center for Extremophile,

Rikkyo University, 3-34-1 Nishiikebukuro,

Toshima-ku, Tokyo 171-8501, Japan

e-mail: tsune@rikkyo.ne.jp 